\title{
Focal Adhesions: A Nexus for Intracellular Signaling and Cytoskeletal Dynamics
}

\author{
Sarita K. Sastry ${ }^{1}$ and Keith Burridge \\ Department of Cell Biology and Anatomy and Lineberger Comprehensive Cancer Center, \\ University of North Carolina, Chapel Hill, North Carolina 27599
}

\section{INTRODUCTION}

Focal adhesions (FAs) are specialized sites of cell attachment to the extracellular matrix (ECM) where integrin receptors link the ECM to the actin cytoskeleton. Integrins cluster into supramolecular complexes with structural, cytoskeletal proteins like talin, vinculin, and $\alpha$-actinin, as well as numerous signaling molecules, including c-Src, FAK, p130cas, and paxillin [1]. The composition and molecular architecture of FAs have been reviewed elsewhere [2-4] and are beyond the scope of this brief review. FAs serve at least two significant cellular functions: to transmit force or tension at adhesion sites to maintain strong attachments to the underlying ECM and to act as signaling centers from which numerous intracellular pathways emanate to regulate cell growth, survival, and gene expression $[5,6]$.

F As are dynamic structures that assemble, disperse, and recycle (turnover) as cells migrate or enter into mitosis. Recent evidence reveals the complexity of these processes. Assembly/disassembly involves the coordinate regulation of Rho family GTPases through cross talk between integrins and numerous adhesion receptors (cadherins, cell adhesion molecules (CAMs), selectins, and syndecans), G-protein-coupled receptors (GPCRs), and receptor tyrosine kinases (RTKs), as well as the interplay between microtubules and actin. It is also apparent that FAs are themselves motile and heterogeneous in composition. Finally, turnover of FAs entails communication with components of vesicle trafficking pathways and microtubules. This review highlights recent findings relating to $\mathrm{FA}$ assembly, dynamics, and turnover.

\footnotetext{
${ }^{1}$ To whom reprint requests should be addressed. Fax: (919) 9661856. E-mail: sksastry@med.unc.edu.
}

\section{FOCAL ADHESION ASSEMBLY}

\section{Contractility}

FAs have long served as a model system for the study of cell-matrix interactions. These structures are prominent in many adherent cell types grown in culture, but are rarely observed in vivo. Several features of the tissue culture environment promote FA assembly [1]. FAs form during spreading or migration on flat, rigid substrates to which ECM components become adsorbed. The assembly of FAs in response to adhesion to the ECM is gradual, usually occurring within 1 to $2 \mathrm{~h}$ after cell attachment. I nitially, nascent cell-matrix adhesions, or focal complexes, form at the cell periphery as a cell spreads or at the leading edge as a cell migrates. Focal complexes mature into FAs as cells become stably attached to their substrates and tension is exerted on these sites of adhesion. Actin filaments are indirectly tethered to integrins at FAs [4, 7]. In migrating cells, FAs can provide traction on the substrate over which cells crawl, although some cells can migrate without FAs and large FAs retard motility due to excessive adhesion [8].

Additionally, cells in culture are grown under conditions that mimic a wound environment, frequently in the presence of serum factors such as Iysophosphatidic acid (LPA). Normally, LPA is secreted into a wound to stimulate cell contraction, contributing to wound closure. However, in tissue culture, contraction is opposed by adhesion to a rigid substrate. This generates isometric tension between sites of strongest adhesion. In turn, the isometric tension results in alignment of bundles of actin filaments (stress fibers) and the clustering of integrins, giving rise to FAs (Fig. 1). An experimental system often utilized to study FA formation uses nonmigratory, adherent fibroblasts that have become quiescent as a result of being serum-starved. Under this condition, FAs and stress fibers are disassembled, despite contact with the underlying ECM. Quiescent 
inhibit cell contractility as evidenced by a decrease in wrinkling of silicone rubber substrates upon which cells had been cultured [19]. In parallel, there was a decrease in the size and number of FAs. Finally, caldesmon overexpression resulted in increased cell spreading and membrane extensions, also a sign of decreased tension. Caldesmon acts downstream of RhoA, since it blocked FA assembly when coexpressed with activated forms of RhoA. It will be interesting to determine the physiological conditions under which caldesmon functions to affect FA assembly.

\section{Activation of RhoA}

Progress has also been made regarding the activation of RhoA. Early work suggested that a tyrosine kinase acted upstream of RhoA and was essential for FA assembly [20]. The identity of this kinase remains elusive. It is clear, however, that many factors can regulate RhoA activity, including integrin signaling, other adhesion receptors, soluble factors like LPA, receptor tyrosine kinase signaling, and components of the microtubule cytoskel eton (Fig. 2) [10, 21]. Using an affinity precipitation assay to directly measure RhoA activity, Ren et al. observed that integrin-mediated adhesion leads to a biphasic response in RhoA activity [22]. Attachment to ECM initially suppresses RhoA activity and this is followed by a modest activation phase. This activation is enhanced significantly in the presence of LPA. Thus LPA is a more potent stimulator of RhoA than is integrin-mediated adhesion to the ECM. This study also showed that adhesion is required to attenuate LPA-induced RhoA activity, since in suspended cells, LPA stimulation led to sustained RhoA activity.

Microtubule depolymerization was shown many years ago to stimulate cell contractility and the assembly of stress fibers [23]. Not surprisingly, subsequent work demonstrated that microtubule depolymerization also resulted in the assembly of FAs [24-28]. Microtubule depolymerization was shown to el evate the level of myosin light chain phosphorylation [29], whereas the formation of stress fibers and FAs was blocked by the inhibitor of RhoA, Botulinum C3 exoenzyme [26, 28]. These findings suggested that microtubule depolymerization stimulates increased actin-myosin contractility by activating RhoA. This was confirmed by direct measurement of RhoA activity [22].

The signal transduction pathways leading to RhoA activation by LPA, integrins, RTKS, or microtubule depolymerization are intense areas of investigation. A schematic diagram of proposed pathways regulating RhoA activation is shown in Fig. 2. The immediate upstream activators of RhoA are guanine nucleotide exchange factors (GEFs), which promote the exchange of GDP for GTP, thus inducing an active conformation of RhoA (or other family members) [30, 31]. The pathway by which LPA elevates RhoA-GTP levels may be the best characterized at present. LPA binds to a heptahelical G-protein-coupled receptor and activates $\mathrm{G} \alpha 12 / 13$ or $\mathrm{G} \beta \gamma$ subunits, which then associate with GEFs for RhoA. Introduction of $\mathrm{G} \alpha 12 / 13$ or $\mathrm{G} \beta \gamma$ subunits into quiescent fibroblasts on their own stimulates FA assembly [32, 33]. In the case of $\mathrm{G} \alpha 12 / 13$ subunits, p115 RhoGEF [34] or PDZ RhoGEF [35] appear to be direct targets. It has also been suggested that the tyrosine kinase PYK2 (CAK $\beta$, CADTK, RAFTK) may play a role in GPCR signaling to RhoA [36].

The regulation of RhoA activity by integrins is complex. Integrins can either stimulate or inhibit RhoA activity depending on the cell type, engagement of specific integrins, and time course of engagement [22, 37, 38]. This duality may reflect the role of integrin-mediated signals in promoting membrane extensions, a condition in which low RhoA activity is desirable, versus the role of integrins in establishing strong attachments across which tension is transmitted, for which higher RhoA activity is needed. As discussed above, in fibroblasts, integrin engagement initially inhibits RhoA activity but later activates it, correlating with the completion of cell spreading [22], during which time integrin-mediated activation of Racl is high [39]. Barry and colleagues found that addition of RGD peptides to quiescent fibroblasts stimulated FA and stress fiber assembly [11]. In adenocarcinoma cells, which are epithelial in origin, crosslinking of $\alpha 6 \beta 4$ integrin stimulates RhoA activity, whereas crosslinking of $\beta 1$ integrins inhibits RhoA [37]. Arthur and colleagues identified a pathway by which integrin engagement results initially in a decrease in RhoA activity [38]. It was found that incubation of fibroblasts with integrin ligands, such as RGD peptides, caused a rapid drop in RhoA activity, but that this did not occur in cells deficient in the Src family tyrosine kinases (Src, Fyn, and Yes). This integrin-mediated drop in RhoA activity was restored if c-Src was reexpressed in these cells. Downstream from c-Src, p190RhoGAP was identified as a target that is phosphorylated and activated, in re sponse to integrin engagement. Interestingly, v-Src expression in fibroblasts has long been known to disrupt FAs and stress fibers (see below).

The pathway by which integrin-mediated adhesion results in long-term activation of RhoA has not been determined. It seems likely that the initial activation of p190RhoGAP must be switched off, but whether there is an additional activation of a RhoA-specific GEF remains to be elucidated. Thus far, numerous GE Fs for the Rho family of GTPases have been discov- 
ered $[30,31,40,41]$ but little information exists concerning how these GEF s become activated by integrin signaling. One exception is Vav1, a hematopoietic GEF for Rho family GTPases. Vav1 is tyrosine phosphorylated and activated in response to integrin engagement or clustering [42-45].

Similar to integrins, receptor tyrosine kinases transmit both positive and negative signals to RhoA. I nitial stimulation (within minutes) with growth factors such as PDGF or EGF promotes the formation of membrane extensions through activation of Racl while suppressing RhoA [46]. RhoA is likely inhibited via Src-dependent activation of p190 RhoGAP [47]. Furthermore, recruitment of p190 RhoGAP to RTKs may be regulated by PYK 2 [48]. Prolonged addition of PDGF (more than $2 \mathrm{~h}$ ) results in FA assembly, suggesting that growth factors can activate RhoA. Recently, a GEF that may mediate growth factor-dependent regulation of RhoA has been identified. Vav2, a widely expressed relative of Vav1, is tyrosine phosphorylated and activated in response to EGF or PDGF stimulation [4951]. Vav2 activates several Rho family members, including Racl and RhoA [49-53].

Finally, how might depolymerization of microtubules lead to enhanced RhoA activity? It has been suggested that intact microtubules may sequester GE F s for RhoA that are released upon microtubule depolymerization [26]. In support of this idea, several GEFs have been found to bind tubulin or microtubules. For example, in hematopoietic cells the exchange factor Vav1 binds tubulin, although whether this affects GEF activity has not been determined [54]. The association of more widely expressed Vav family members, Vav2 or Vav3, with microtubules has not been fully investigated. GEFH 1, which is specific for RhoA, has recently been shown to bind microtubules [55]. The existence of multiple GE Fs for RhoA reflects either a functional redundancy or that specific GEF s act on RhoA in response to distinct stimuli.

Although integrins and GPCRs are a major focus in upstream regulation of RhoA, other adhesion receptors also promote FA assembly, likely via the activation of RhoA. Recently, a role for syndecans in FA assembly has been demonstrated. Syndecan-4 is a transmembrane member of the heparan sulfate proteoglycan family that localizes in FAs [56]. Its overexpression in cultured fibroblasts increases FAs and stress fibers [57, 58], whereas syndecan-4 null cells have impaired FAs [59]. Additionally, FA assembly on the cell binding domain of fibronectin is promoted by antibody ligation of syndecan-4 in a RhoA-dependent manner [60]. A particularly intriguing system in which multiple adhe sion receptors, including selectins, integrins, and other CAMs, cooperate to potentially regulate RhoA is the interaction of leukocytes with endothelial cells during inflammation. Adhesion of monocytes to endothelial cells induces the assembly of stress fibers and presumably FAs in the endothelial cells. Crosslinking of the endothelial cell adhesion molecules VCAM-1, ICAM-1, or E-selectin, but not ICAM-2 or ICAM-3, also stimulates stress fiber formation [61]. It will be important to determine whether these effects are due to increased RhoA activity. In contrast to the above-mentioned cell adhesion molecules, the crosslinking of which promotes FA assembly, the formation of adherens junctions of the cadherin type tends to inhibit FA assembly. The mechanism of this inhibition has not been determined. Recent work, however, has identified a cadherin-binding protein, p120 catenin, as a regulator of Rho family GTPases $[62,63]$. Overexpression of p120 catenin disrupts focal adhesions and stress fibers and decreases RhoA activity in cells. In addition, p120 cate nin elevates the activity of Racl and Cdc42 and binds to Vav2, a Rho family GEF [62].

\section{FOCAL ADHESION DISASSEMBLY}

FAs disassemble or disperse under a number of physiological situations. For example, adhesions to the ECM are released at the rear of a migrating cell and this is accompanied by a disruption of FAs. Another instance of FA disassembly occurs during mitosis, during which cells lose their attachments to the ECM and adopt a round morphology. Finally, in oncogenically transformed fibroblasts, FA integrity is often compromised [64]. Since FA assembly involves both the activation of RhoA and the stimulation of contractility, loss of FAs would ostensibly involve mechanisms that counteract these pathways. The role of integrin signaling in FA disassembly has recently been reviewed [65]. Here we highlight some novel and significant observations.

\section{GAPs}

GTPase-activating proteins, or GAPs, which promote hydrolysis of GTP to GDP, are immediate upstream inhibitors of RhoA. Indeed, introduction of several GAPs into cultured cells leads to a loss of FAs and actin stress fibers and also causes cell rounding. As discussed above, p190RhoGAP suppresses RhoA activity in response to integrin ligation [38] as well as growth factor stimulation [47]. Other GAPs for RhoA which may participate in FA disassembly have also been identified, including GRAF , or GAP for Rho associated with FAK [66], and p122GAP, which blocks LPA-induced FA and stress fiber assembly [67]. I nterestingly, a novel RhoGAP that acts on RhoA in mitotic cells has recently been described [68]. 
Interplay of Rho and Ras Family GTPases

Our understanding of FA disassembly is further complicated by recent evidence that additional Rho family GTPases can affect FA organization. It has long been noted phenomenologically that the actions of Rac and RhoA are functionally antagonistic. Racl promotes membrane extension, whereas in many situations RhoA induces membrane retraction. Such a reciprocal relationship is most clearly seen in migrating fibroblasts or in growth cones of neurons. In epithelial cells, activation of Rac promotes the assembly of cell-cell junctions and blocks FA formation, while activation of RhoA promotes a fibroblastic phenotype [46, 69-71]. Rottner et al. have recently shown that expression of a dominant negative Racl mutant in quiescent fibroblasts induces FA assembly [72]. This effect could be attributed to regulation of contractility by Racl effectors downstream of RhoA [73]. However, using affinity precipitation assays to measure RhoA activity, Sander et al. have shown that activation of Racl can itself suppress RhoA activity [46]. This was shown either by expression of TIAM-1, an exchange factor for Rac1, or by expression of activated mutants of Racl in NIH3T3 fibroblasts. Cdc42 expression also antagonized RhoA activity. The mechanism by which Racl (or Cdc42) inhibits RhoA is currently unknown. A signaling complex that links Racl effectors to GAPs for RhoA would be one potential mechanism.

A newly identified Rho family member, RhoE/rnd, also disrupts $F$ As and stress fibers $[74,75]$. RhoE/rnd was identified as a binding partner for p190RhoGAP [76]. Unlike RhoA, which cycles between GDP- and GTP-bound states, RhoE is constitutively active in the GTP-bound state and is insensitive to GAPs. It is unclear how RhoE functions. One possibility is that it titrates away downstream effectors from RhoA. It is also possible that RhoE can inhibit RhoA activity, although this has not been directly demonstrated. The function of RhoE within cells remains undetermined.

Finally, the Ras pathway is suggested to play a role in FA turnover. Active Ras is required for the turnover of FAs during cell migration [77]. Interestingly, the duration of Ras activation affects the activation state of RhoA. Transient expression of activated Ras in epithelial cells results in activation of Racl and inhibition of RhoA activity [71]. In contrast, sustained Ras activation promotes enhanced RhoA activity [71] as well as a fibroblastic morphology [70, 71]. Knockout cell lines lacking p120 RasGAP, an upstream inhibitor of Ras, are unable to adopt a polarized morphology [78] or turn over FAs. This effect of p120 RasGAP is independent of Ras regulation. Instead, p120 RasGAP regulates cell polarity, and presumably FA turnover, via its interaction with p190 RhoGAP.
Tyrosine Phosphorylation

As mentioned earlier, signaling from a tyrosine kinase is necessary for FA assembly. It was thought that Src family kinases or perhaps FAK may be required for FAs to form. However, through the analysis of knockout cell lines, it is apparent that Src family kinases play a role in FA disassembly or turnover [80]. As discussed above, c-Src is required for inhibition of RhoA by integrins [38]. Furthermore, overexpression of $\mathrm{V}$-Src leads to FA disruption, while the expression of kinase-inactive $v$-Src in normal cells leads to formation of exaggerated FAs [81]. FAK is also likely to play a role in FA turnover or disassembly. FAK-null cells possess abnormally large FAs and are unable to migrate [82]. In contrast, overexpression of FAK stimulates motility [83]. Consistent with this finding is the observation that in permeabilized fibroblasts, an increase in tyrosine phosphorylation accompanies FA disruption in response to ATP [79].

Significant progress has also been made in identifying protein tyrosine phosphatases that may promote FA disassembly or turnover. Knockout cell lines of either SHP-2 or PTP-PEST exhibit enhanced FAs [84, 85]. The mechanism of this phenotype remains to be determined. However, one or more PTPases may act upstream of RhoA. Using calpeptin, an inhibitor originally designed for the $\mathrm{Ca}^{2+}$-dependent protease calpain, it was recently demonstrated that this inhibitor stimulates FA assembly in quiescent fibroblasts through inhibition of a PTPase [86]. Calpeptin-induced F A assembly was blocked by C3 exoenzyme, indicating that the PTPase acts upstream of RhoA. In addition two transmembrane PTPases, LAR and RPTP $\alpha$, have been shown to localize in FAs under restricted conditions $[87,88]$. Finally, PTPases may play a major role in regulation of $\mathrm{FA}$ disassembly during mitosis. Comparison of interphase versus mitotic cells shows that FAK, p130cas, and paxillin are dephosphorylated on tyrosine residues but phosphorylated on serine and threonine in mitosis [89]. These proteins are rapidly tyrosine phosphorylated in response to adhesion [3, 5, 18]. In mitotic extracts, FAK kinase activity is decreased and its associations with p130cas and paxillin are disrupted. The disruption of this complex is thought to prevent integrin signaling until the completion of cytokinesis. It will be interesting to determine which tyrosine phosphatases and which serine-threonine kinases act on these proteins in mitosis.

\section{HETEROGENEITY OF CELL-MATRIX ADHESIONS}

The molecular architecture of FAs and the nature of specific protein interactions have been reviewed elsewhere [1-4] and will not be dealt with in detail here. It 
is noteworthy, however, that recent observations point to considerable molecular and structural diversity among FAs in a single cell and also within individual FAs. Using fluorescence ratio imaging, the distribution of several FA proteins was compared. This analysis has identified at least three structurally distinct types of adhesion sites whose molecular compositions differ. Classical FAs are large, spearheaded or ellipsoid in shape, and located at the cell periphery and contain vinculin, paxillin, phosphotyrosine, and $\alpha \mathrm{v} \beta 3$ integrins [90]. In contrast, fibrillar adhesions are el ongated, centrally located, and contain tensin, $\alpha 5 \beta 1$ integrins, and fibronectin with little or no phosphotyrosine, vinculin, or paxillin. Finally, "mosaic" FAs are morphologically similar to "classical" FAs, but their content is variable. Interestingly, the assembly of these distinct adhesions depends on several critical factors. The first is contractility. The assembly of classical FAs but not fibrillar adhesions was sensitive to contractility inhibitors [90]. The second is the physical state of the ECM. A nonimmobilized fibronectin matrix that is adsorbed to the substrate promotes fibrillar adhesions, while an immobilized matrix, crosslinked to the substrate, leads to the formation of classical FAs [91]. The third factor is the type of integrin involved. Classical FAs typically contain $\alpha \mathrm{v} \beta 3$ integrins and fibrillar adhesions contain $\alpha 5 \beta 1$ [90]. Using GFP-tagged c-Src, Felsenfeld and coworkers noted that c-Src selectively localizes in phosphotyrosine-rich FAs formed by $\alpha \vee \beta 3$ integrins, but not those formed by $\alpha 5 \beta 1$ integrins [92]. This type of analysis has been performed only on some FA components. A more comprehensive survey will be informative and may reveal additional complexities.

\section{TURNOVER AND DYNAMICS OF FOCAL ADHESIONS}

Up to this point, much of this review has dealt with potential pathways leading to FA formation or disassembly. These are active, dynamic processes intimately associated with turnover or recycling of FAs. Information from knockout cell lines and biochemical screening has led to the identification of potential regulators of FA turnover. Additionally, the use of elegant imaging techniques to visualize cell-substrate contact dynamics in live cells has contributed to our knowledge of how FAs undergo remodeling and turnover during cell spreading and motility.

\section{Movement of Focal Adhesions}

FAs that form at the front of a migrating cell generally remain fixed relative to the substrate as the cell moves over them. FAs then disperse at the cell tail [93, 94]. Stationary FAs maintain stable attachments to the ECM to resist actin-myosin contraction that propels the cell forward. However, in some situations FAs move relative to the substrate. A recent study found that static FAs occur primarily in motile cells. Using GFP-tagged $\beta 1$ integrin subunits, Smilenov et al. compared FA movement in stationary (nonmotile) and migrating fibroblasts [95]. Using time-lapse imaging of live cells and overlaying of sequential images, they found that FAs in nonmotile cells are not static. Instead, FAs in stationary cells were observed to move linearly toward the cell center. This movement depended on actin-myosin contractility since FA move ment was not observed in the presence of BDM, a myosin inhibitor. Microtubule disassembly, which en-

syndecan-4 stimulates focal adhesion and stress fiber assembly likely through activation of RhoA (dotted arrows). The formation of adherens junctions, containing cadherins, antagonizes focal adhesion and stress fiber formation, possibly via pl20 catenin, whose overexpression inhibits RhoA activity. Finally, integrins transmit both positive and negative signals to RhoA. I nitially, engagement of integrins with ECM inhibits RhoA. Integrins activate p190 RhoGAP through a c-Src-dependent mechanism. I ntegrins also activate Racl as a cell spreads, which can antagonize RhoA activity. As stable adhesions form, integrins activate RhoA, most likely through an integrin-dependent Rho GEF. Downstream of RhoA, actin-myosin contractility stimulates actin stress fiber formation and clustering of integrins and associated proteins to form focal adhesions. Actin-myosin contractility is positively regulated by RhoA effectors, like Rho kinase, and negatively regulated by caldesmon, an actin-binding protein.

FIG. 3. Focal adhesion (FA) dynamics and turnover in nonmotile and motile fibroblasts is partly controlled by cross talk between adhesion sites and the microtubule cytoskeleton. (A) A nonmotile, unpolarized fibroblast. (I) FA movement, relative to the substrate, toward the cell center depends on actin-myosin contractility. Stress fibers under isometric tension contract, pulling FAs centripetally inward. (II) Growing microtubules target FAs. FAs capture and stabilize microtubules by capping microtubule ends. (IIII) Microtubule depolymerization enhances FA assembly through activation of RhoA. FA movement toward the cell center is enhanced due to increased actin-myosin contractility. (IV) Active assembly of microtubules induces FA disassembly and turnover. Microtubules deliver local "relaxation" signals to FAs; presumably these factors regulate RhoA activity or contractility downstream of RhoA. (B) In a polarized, motile fibroblast, FA movement is generally not observed but FAs have distinct behaviors in different regions of the cell. (I) Formation zone: nascent FA (focal complexes) form at the leading edge. Microtubule polymerization activates Racl and stimulates membrane protrusions. Growing microtubules target selected FAs at the leading edge and induce their disassembly. (II) Persistence zone: a fraction of nascent FAs matures into stable, static FAs in which contractile stress fibers exert tension on the substrate. Capture and capping of microtubules by FAs leads to increased FA stability. (III) Culling zone: growth of microtubules toward FAs at the cell rear promotes FA disassembly and turnover. (IV) Motile zone: contractility during tail retraction may cause some FAs at the tail to move relative to the substrate toward the cell center. UItimately, these posterior FAs release from the substrate and disassemble. 


\section{hances RhoA activity [22] and contractility [23, 29],} increased the rate of FA movement. Interestingly, although FA movement was typically not observed in migrating cells, distinct zones of $\mathrm{FA}$ behavior were discerned. At the leading edge of a migrating cell is a formation zone; between the leading edge and the nucleus, a persistence zone exists in which stable FAs continue to grow and mature; between the nucleus and the tail a culling zone exists, where FAs turn over; at the cell tail is a small motile zone [95]. The different behaviors of $\mathrm{FAs}$ in both nonmigratory fibroblasts and polarized, migrating fibroblasts are depicted in Fig. 3. These findings have led to a molecular clutch model in which FAs transition between a motile and a nonmotile state in a contractility-dependent manner, to balance adhesive forces and migratory cues. How universal this behavior of FAs is remains to be determined.

\section{Cross Talk between Microtubules and Focal Adhesions}

The state of the microtubule cytoskel eton can greatly influence the organization of FAs and actin stress fibers. An emerging view is that the relationship be tween microtubules and FAs is reciprocal, in which the organization of one affects the dynamics of the other (Fig. 3). As mentioned earlier, disruption of microtubules activates RhoA, leading to increased actin-myosin contractility, FAs, and stress fibers [23-29]. Conversely, elevated RhoA activity stabilizes microtubules [96]. In addition, Waterman-Storer and co-workers found that active microtubule polymerization was associated with increased Racl activity and membrane protrusions [97]. Given the antagonism between Racl and RhoA, these findings suggest that sites of microtubule growth would be associated with locally high concentrations of active Racl and decreased RhoA activity. Consequently, microtubule growth would be expected to promote focal adhesion disassembly. I ndeed, Small and co-workers found that microtubule polymerization is associated with local destabilization of focal adhesions [98]. Small and his colleagues noted earlier that there is an association between the ends of microtubules and focal adhesions [98-100] and they speculated that this association might stabilize the adhesions. More recent work from this group, however, has shown that this association is antagonistic: the targeting of microtubules to focal adhesions causes the disassembly of these structures [101]. These investigators propose that growing microtubules negatively regulate focal adhesions by delivering a localized relaxing signal to this region. The evidence points to microtubule dynamics regulating the activity of Rho family GTPases in very localized regions of the cytoplasm, but proof of this awaits assays that will reveal the activity of these Rho family proteins at a subcellular level.

\section{Delivery of Components to Focal Adhesions}

An emerging concept is that some focal adhesion proteins are actively targeted to and from focal adhe sions via a vesicle trafficking pathway. This also likely involves microtubules and may be another way in which microtubule behavior affects focal adhesion turnover. Several studies have implicated the ARF (ADP-ribosylation factor) family of GTPases in this targeting. ARFs have been shown to control intracellular membrane trafficking, including the delivery of membrane to sites of membrane protrusion [102]. They have also been shown to have a role in regulating cytoskeletal organization through an interplay with Rho family GTPases [103, 104]. ARF 1 activity has been shown to be required for the recruitment of paxillin from the perinuclear compartment to focal adhesions [103]. Like Rho family GTPases, ARF s cycle between an inactive GDP-bound state and an active GTP-bound state. The mechanism by which paxillin is recruited to focal adhesions may lie in its interactions with ARF GAPs. Paxillin has been shown to associate with a 95-kDa ARF-GAP, variously named PKL [105], PAG3/ PAP $\alpha$ [106], or GIT [107]. This ARF-GAP acts on ARF 6 [106] and is localized to focal adhesions [105]. Kondo et al. found that the activity of this ARF-GAP prevents paxillin recruitment to focal adhesions [106]. Another protein that is recruited to focal adhesions from the perinuclear regions is v-Src [108]. However, recruitment of v-Srcto F As does not require its kinase activity [81]. Whether this recruitment involves ARF activity has not yet been determined. An intriguing connection between ARFs and Src is suggested by the finding that ASAP-1, another ARF-GAP that localizes to FAs [109], is phosphorylated by Src family kinases [110]. Finally, some integrins associate with ARF-GEFs. For example, $\beta 2$ integrin cytoplasmic domains associate with cytohesin-1, an ARF-GEF, and this interaction is implicated in the regulation of integrin affinity [111, 112] The roles of ARFs and their regulatory GAPs and GEFs in focal adhesion turnover are only just beginning to be discovered. This promises to be an exciting area in the future.

\section{CONCLUDING REMARKS}

In this brief review of focal adhesions, we have tried to emphasize some of the recent developments. We anticipate that a number of emerging areas will continue to be developed in the next few years. The idea that FAs are heterogeneous bears further investigation. The relative temporal and spatial distributions of 
many components have yet to be compared. The role of ARFs, along with their regulatory GEFs and GAPs, is currently enigmatic. The evidence for cross talk between ARFs and Rho family proteins is particularly intriguing. A great many factors influence the activity of Rho family proteins. In general, the effects of agents that stimulate or inhibit RhoA activity have been measured on whole populations of cells. In many situations, however, very localized, subcellular changes in RhoA activity are likely to be important. This is suggested by the studies on microtubule dynamics. The evidence indicates that the behavior of individual $\mathrm{mi}$ crotubules regulates the local activity of RhoA or Racl, thereby affecting $\mathrm{FA}$ assembly and turnover in specific regions of the cell. The development of methods to determine subcellular changes in activity of Rho family proteins should yield a greater understanding of how various factors control FA dynamics.

We thank Dr. Becky Worthylake, Bill Arthur, and Betty P. Liu for critical review of the manuscript and for helpful discussions. We apol ogize to those individuals whose work we were unable to cite due to space limitations. This work was supported by NIH Grants GM 29860 and HL45100 (Keith Burridge).

\section{REFERENCES}

1. Burridge, K., and Chrzanowska-Wodnicka, M. (1996). Focal adhesions, contractility, and signaling. Annu. Rev. Cell Dev. Biol. 12, 463-519.

2. J ockusch, B., Bubeck, P., Giehl, K., Kroemker, M., Moschner, J ., Rothkegel, M., Rudiger, M., Schluter, K., Stanke, G., and Winkler, J. (1995). The molecular architecture of focal adhesions. Annu. Rev. Cell Dev. Biol. 11, 379-416.

3. Yamada, K., and Geiger, B. (1997). Molecular interactions in cell adhesion complexes. Curr. Opin. Cell Biol. 9, 76-85.

4. Critchley, D. (2000). Focal adhesions-The cytoskeletal connection. Curr. Opin. Cell Biol. 12, 133-139.

5. Schwartz, M., Schaller, M., and Ginsberg, M. (1995). Integrins: Emerging paradigms of signal transduction. Annu. Rev. Cell Biol. 11, 549-599.

6. Howe, A., Aplin, A., Alahari, S., and J uliano, R. L. (1998). Integrin signaling and cell growth control. Curr. Opin. Cell Biol. 10, 220-231.

7. Calderwood, D., Shattil, S., and Ginsberg, M. (2000). Integrins and actin filaments: Reciprocal regulation of cell adhesion and signaling. J . Biol. Chem. 275, 22607-22610.

8. Huttenlocher, A., Sandborg, R., and Horwitz, A. F. (1995). Adhesion in cell migration. Curr. Opin. Cell Biol. 7, 697-706.

9. Ridley, A., and Hall, A. (1992). The small GTP-binding protein Rho regulates the assembly of focal adhesions and actin stress fibers in response to growth factors. Cell 70, 389-399.

10. Hall, A. (1998). Rho GTPases and the actin cytoskeleton. Science 279, 509-514.

11. Barry, S. T., Flinn, H. M., Humphries, M. J ., Critchley, D. R., and Ridley, A. J. (1996). Requirement for Rho in integrin signalling. Cell Adhes. Commun. 4, 387-398.

12. Hotchin, N. A., and Hall, A. (1995). The assembly of integrin adhesion complexes requires both extracellular matrix and intracellular Rho/Rac GTPases. J . Cell Biol. 131, 1857-1865.
13. Clark, E., King, W., Brugge, J ., Symons, M., and Hynes, R. (1998). Integrin-mediated signals regulated by members of the Rho family of GTPases. J . Cell Biol. 142, 573-586.

14. Chrzanowska-Wodnicka, M., and Burridge, K. (1996). Rhostimulated contractility drives the formation of stress fibers and focal adhesions. J. Cell Biol. 133, 1403-1415.

15. Kaibuchi, K., Kuroda, S., and Amano, M. (1999). Regulation of the cytoskel eton and cell adhesion by the Rho family GTPases in mammalian cells. Annu. Rev. Biochem. 68, 459- 486.

16. Amano, M., Ito, M., Kimura, K., Fukata, Y., Chihara, K., Nakano, T., Matsuura, Y., and Kaibuchi, K. (1996). Phosphorylation and activation of myosin by Rho-associated kinase (Rho-kinase). J . Biol. Chem. 271, 20246-20249.

17. Kimura, K., Ito, M., Amano, M., Chihara, K., Fukata, Y., Nakafuku, M., Yamamori, B., Feng, J ., Nakano, T., Okawa, K., I wamatsu, A., and Kaibuchi, K. (1996). Regulation of myosin phosphatase by Rho and Rho-associated kinase (Rho-kinase). Science 273, 245-248.

18. Burridge, K., Chrzanowska-Wodnicka, M., and Zhong, C. (1997). Focal adhesion assembly. Trends Cell Biol. 7, 342-347.

19. Helfman, D., Levy, E., Berthier, C., Shtutman, M., Riveline, D., Grosheva, I., Lachish-Zalait, A., Elbaum, M., and Bershadsky, A. (1999). Caldesmon inhibits nonmuscle cell contractility and interferes with the formation of focal adhesions. Mol. Biol. Cell 10, 3097-3112.

20. Ridley, A., and Hall, A. (1994). Signal transduction pathways regulating Rho-mediated stress fiber formation: Requirement for a tyrosine kinase. EMBO J . 13, 2600-2610.

21. Schwartz, M., and Shattil, S. (2000). Signaling networks linking integrins and Rho family GTPases. Trends Biochem. Sci. 25, 388-391.

22. Ren, X., Kiosses, W., and Schwartz, M. (1999). Regulation of the small GTP-binding protein Rho by cell adhesion and the cytoskeleton. EMBO J . 18, 578-585.

23. Danowski, B. (1989). Fibroblast contractility and actin reorganization are stimulated by microtubule inhibitors. J . Cell Sci. 93, 255-266.

24. Bershadsky, A., Chausovsky, A., Becker, E., Lyubimova, A., and Geiger, B. (1996). Involvement of microtubules in the control of adhesion-dependent signal transduction. Curr. Biol. 6, 1279-1389.

25. Enomoto, T. (1996). Microtubule disruption induces the formation of actin stress fibers and focal adhesions in cultured cells: Possible involvement of the Rho signal cascade. Cell Struct. Funct. 2, 317-326.

26. Liu, B. P., Chrzanowska-Wodnicka, M., and Burridge, K. (1998). Microtubule depolymerization induces stress fibers, focal adhesions, and DNA synthesis via the GTP-binding protein Rho. Cell Adhes. Commun. 5, 249-255.

27. Pletjushkina, O., Belkin, A., I vanova, O., Oliver, T., J acobson, K., and Vasiliev, J. (1998). Maturation of cell-substratum focal adhesions induced by depolymerization of microtubules is induced by increased cortical tension. Cell Adhes. Commun. 5, 121-135.

28. Zhang, Q., Magnusson, M., and Mosher, D. (1997). Lysophosphatidic acid and microtubule-destabilizing agents stimulate fibronectin matrix assembly through Rho-dependent actin stress fiber formation and cell contraction. Mol. Biol. Cell 8, 1415-1425.

29. Kolodney, M., and Elson, E. (1995). Contraction due to micro tubule disruption is associated with increased phosphorylation of myosin regulatory light chain. Proc. Natl. Acad. Sci. USA 92, 10252-10256. 
30. Van Aelst, L., and D'Sousa-Schorey, C. (1997). Rho GTPases and signaling networks. Genes Dev. 11, 2295-2322.

31. Whitehead, I., Campbell, S., Rossman, K., and Der, C. (1997). Dbl family proteins. Biochim. Biophys. Acta 1332, F1-F23.

32. Buhl, A., J ohnson, N., Dhanasekaran, N., and J ohnson, G. (1995). $G \alpha 12$ and $G \alpha 13$ stimulate Rho-dependent stress fiber formation and focal adhesion assembly. J . Biol. Chem. 270, 24631-24634.

33. Ueda, H., Itoh, H., Yamauchi, J., Morishita, R., Kaziro, Y., Kato, K., and Asano, T. (2000). G protein $\beta \gamma$ subunits induce stress fiber formation and focal adhesion assembly in a Rhodependent manner in HeLa cells. J . Biol. Chem. 275, 20982102.

34. Hart, M., Jiang, X., Kozasa, T., Roscoe, W., Singer, W., Gilman, A., Sternweis, P., and Bollag, G. (1998). Direct stimulation of the guanine nucleotide exchange activity of pl15 RhoGEF by $\mathrm{G} \alpha \mathbf{1 3}$. Science 280, 2112-2114.

35. Fukuhara, S., Murga, C., Zohar, M., I gishi, T., and Gutkind, J . (1999). A novel PDZ domain containing guanine nucleotide exchange factor links heterotrimeric $G$ proteins to Rho. J . Biol . Chem. 274, 5868-5879.

36. Shi, C., Sinnarajah, S., Cho, H., Kozasa, T., and Kehrl, J. (2000). G $\alpha 13$-mediated PYK2 activation: PYK2 is a mediator of $\mathrm{G} 13 \alpha$-induced serum response element dependent transcription. J . Biol. Chem. 275, 24470-24476.

37. O'Connor, K., Ngyuen, B., and Mercurio, A. (2000). RhoA function in lamellae formation and migration is regulated by the $\alpha 6 \beta 4$ integrin and CAMP metabolism. J . Cell Biol. 148, 253258.

38. Arthur, W. T., Petch, L., and Burridge, K. (2000). Integrin engagement suppresses RhoA activity via a c-Src-dependent mechanism. Curr. Biol. 10, 719-722.

39. del Pozo, M., Price, L., Alderson, N., Ren, X., and Schwartz, M. (2000). Adhesion to the extracellular matrix regulates the coupling of the small GTPase Rac to its effector PAK. EMBO J . 19, 2008-2014.

40. Tatsumoto, T., Xie, X., Blumenthal, R., Okamoto, I., and Miki, T. (1999). Human ECT2 is an exchange factor for Rho GTPases, phosphorylated in G2/M phases, and involved in cytokinesis. J. Cell Biol. 147, 921-928.

41. Rumenapp, U., Blomquist, A., Schworer, G., Schablowski, H., Psoma, A., and J akobs, K. (1999). Rho-specific binding and guanine nucleotide exchange catalysis by KIAA0380, a Dbl family member. FEBS Lett. 459, 313-318.

42. Zheng, L., Sjolander, A., Eckerdal, J., and Andersson, T. (1996). Antibody-induced engagement of beta 2 integrins on adherent human neutrophils triggers activation of p21ras through tyrosine phosphorylation of the protooncogene product Vav. Proc. Natl. Acad. Sci. USA 93, 8431- 8436.

43. Yron, I., Deckert, M., Reff, M., Munshi, A., Schwartz, M., and Altman, A. (1999). Integrin-dependent tyrosine phosphorylation and growth regulation by Vav. Cell Adhes. Commun. 7, 1-11.

44. Gotoh, A., Takahira, H., Geahlen, R., and Broxmeyer, H. (1997). Cross-linking of integrins induces tyrosine phosphorylation of the proto-oncogene product Vav and the protein tyrosine kinase Syk in human factor-dependent myeloid cells. Cell Growth Differ. 8, 721-729.

45. Miranti, C. K., Leng, L., Maschberger, P., Brugge, J. S., and Shattil, S. J . (1998). I dentification of a novel integrin signaling pathway involving the kinase Syk and the guanine nucleotide exchange factor Vav1. Curr. Biol. 8, 1289-1299.
46. Sander, E., ten K looster, J ., van Delft, S., van der Kammen, R. and Collard, J . (1999). Rac downregulates Rho activity: Reciprocal balance between both GTPases determines cellular morphology and migratory behavior. J . Cell Biol. 147, 1009-1021.

47. Chang, J ., Gill, S., Settleman, J ., and Parsons, S. (1995). C-Src regulates the simultaneous rearrangement of actin cytoskele ton, p190RhoGAP, and p120RasGAP following epidermal growth factor stimulation. J . Cell Biol. 130, 355-368.

48. Zrihan-Licht, S., Fu, Y., Settleman, J., Schinkmann, K. Shaw, L., Keydar, I., Avraham, S., and Avraham, H. (2000). RAFTK/PYK2 tyrosine kinase mediates the association of p190RhoGAP with RasGAP and is involved in breast cancer cell invasion. Oncogene 19, 1318-1328.

49. Liu, B. P., and Burridge, K. (2000). Vav2 activates Rac1, Cdc42 and RhoA downstream from growth factor receptors but not $\beta 1$ integrins. Mol. Cell. Biol. 20, 7160-7169.

50. Moores, S., Selfors, L., Fredericks, J ., Breit, T., Fujikawa, K., Alt, F., Brugge, J., and Swat, W. (2000). Vav family proteins couple to diverse cell surface receptors. Mol. Cell. Biol. 20, 6364-6373.

51. Pandey, A., Podtelejnikov, A., Blagoev, B., Bustelo, X., Mann, M., and Lodish, H. (2000). Analysis of receptor signaling pathways by mass spectrometry: Identification of Vav-2 as a substrate of the epidermal and platelet-derived growth factor receptors. Proc. Natl. Acad. Sci. USA 97, 179-184.

52. Schuebel, K., Movilla, N., Rosa, J., and Bustelo, X. (1998). Phosphorylation-dependent and constitutive activation of Rho proteins by wild-type and oncogenic Vav-2. EMBO J. 16, 6608-6621.

53. Abe, K., Rossman, K., Liu, B. P., Ritola, K., Chiang, D., Campbell, S., Burridge, K., and Der, C. (2000). Vav2 is an activator of Cdc42, Racl, and RhoA. J . Biol. Chem. 275, 10141-10149.

54. Fernandez, J ., Keshvara, L., Peters, J ., furlong, M., Harrison, M., and Geahlen, R. (1999). Phosphorylation- and activationindependent association of the tyrosine kinase substrates $\mathrm{Cbl}$ and Vav with tubulin in B-cells. J . Biol. Chem. 274, 14011406.

55. Ren, Y., Li, R., Zheng, Y., and Busch, H. (1998). Cloning and characterization of GEF-H 1, a microtubule-associated guanine nucleotide exchange factor for Rac and Rho GTPases. J . Biol. Chem. 273, 34954-34960.

56. Couchman, J., and Woods, A. (1999). Syndecan-4 and integrins: Combinatorial signaling in cell adhesion. J. Cell Sci. 112, 3415-3420.

57. Echtermeyer, F., Baciu, P., Saoncella, S., Ge, Y., and Goetinck, P. (1999). Syndecan-4 core protein is sufficient for the assembly of focal adhesions and actin stress fibers. J . Cell Sci. 112, 3433-3441.

58. Longley, R., Woods, A., Fleetwood, A., Cowling, G., Gallagher, J ., and Couchman, J . (1999). Control of morphology, cytoskeleton, and migration by syndecan-4. J . Cell Sci. 112, 34213431.

59. I shiguro, K., Kadomatsu, K., Kojima, T., Muramatsu, H., Tsuzuki, S., Nakamura, E., Kusugami, K., Saito, H., and Muramatsu, T. (2000). Syndecan-4 deficiency impairs focal adhesion formation only under restricted conditions. J . Biol. Chem. 275, 5249-5252.

60. Saoncella, S., Echtermeyer, F., Denhez, F., Nowlen, J., Mosher, D., Robinson, S., Hynes, R., and Goetinck, P. (1999). Syndecan-4 signals cooperatively with integrins in a Rho-dependent manner in the assembly of focal adhesions and actin stress fibers. Proc. Natl. Acad. Sci. USA 96, 2805-2810. 
61. Wojciak-Stothard, B., Williams, L., and Ridley, A. (1999). Monocyte adhesion and spreading on human endothelial cells is dependent on Rho-regulated receptor clustering. J . Cell Biol. 145, 1293-1307.

62. Noren, N., Liu, B., Burridge, K., and Kreft, B. (2000). p120 catenin regulates the actin cytoskeleton via Rho family GTPases. J. Cell Biol. 150, 567-580.

63. Anastasiadis, P., Moon, S., Thoreson, M., Crawford, H., Zheng, Y., and Reynolds, A. (2000). Inhibition of RhoA by p120 catenin. Nature Cell Biol. 2, 637-644.

64. Hynes, R. (1992). Integrins: Versatility, modulation, and signaling in cell adhesion. Cell 69, 11-25.

65. Schoenwaelder, S. M., and Burridge, K. (1999). Bidirectional signaling between the cytoskel eton and integrins. Curr. Opin. Cell Biol. 11, 274-286.

66. Taylor, J., Macklem, M., and Parsons, J. T. (1999). Cytoskeletal changes induced by GRAF, the GTPase regulator associated with focal adhesion kinase, are mediated by Rho. J . Cell Sci. 112, 231-242.

67. Sekimata, M., Kabuyama, Y., Emori, Y., and Homma, Y. (1999). Morphological changes and detachment of adherent cells induced by p122, a GTPase-activating protein for Rho 274, 17757-17762.

68. J antsch-Plunger, V., Gonczy, P., Romano, A., Schnabel, H., Hamill, D., Schnabel, R., Hyman, A., and Glotzer, M. (2000). CYK-4: A Rho family GTPase activating protein (GAP) required for central spindle formation and cytokinesis. J . Cell Biol. 149, 1391-1404.

69. Braga, V., Machesky, L., Hall, A., and Hotchin, N. (1997). The small GTPases Rho and Rac are required for the establishment of cadherin-dependent cell-cell contacts. J. Cell Biol. 137, 1421-1431.

70. Zhong, C., Kinch, S., and Burridge, K. (1997). Rho-stimulated contractility contributes to the fibroblastic phenotype of Rastransformed epithelial cells. Mol. Biol. Cell 8, 2329-2344.

71. Zondag, G., Evers, E., ten Klooster, J ., J anssen, L., van der Kammen, R., and Collard, J . (2000). Oncogenic Ras downregulates Rac activity, which leads to increased Rho activity and epithelial-mesenchymal transition. J . Cell Biol. 149, 775-781.

72. Rottner, K., Hall, A., and Small, J . (1999). Interplay between Rac and Rho in the control of substrate contact dynamics. Curr. Biol. 9, 640-648.

73. Sanders, L., Matsumura, F., Bokoch, G., and de Lanerolle, P. (1999). Inhibition of myosin light chain kinase by p21-activated kinase (PAK). Science 283, 2083-2085.

74. Nobes, C., Lauritzen, I., Mattei, M., Paris, S., Hall, A., and Chardin, P. (1998). A new member of the Rho family, Rnd1, promotes disassembly of actin filament structures and loss of cell adhesion. J. Cell Biol. 141, 187-197.

75. Guasch, R., Scambler, P., J ones, G., and Ridley, A. (1998). RhoE regulates actin cytoskeleton organization and cell migration. Mol. Cell. Biol. 18, 4761-4771.

76. Foster, R., Hu, K., Lu, Y., Nolan, K., Thissen, J ., and Settleman, J . (1996). Identification of a novel human Rho protein with unusual properties: GTPase deficiency and in vivo farnesylation. Mol. Cell. Biol. 16, 2689-2699.

77. Nobes, C., and Hall, A. (1999). Rho GTPases control polarity, protrusion, and adhesion during cell movement. J . Cell Biol. 144, 1235-1244.

78. Kulkarni, S., Gish, G., van der Geer, P., Henkemeyer, M., and Pawson, T. (2000). Role of p120 Ras-GAP in directed cell movement. J . Cell Biol. 149, 457- 470.
79. Crowley, E., and Horwitz, A. F. (1995). Tyrosine phosphorylation and cytoskel etal tension regulate the release of fibroblast adhesions. J . Cell Biol. 131, 525-537.

80. Klinghoffer, R., Sachsenmaier, C., Cooper, J ., and Soriano, P. (1999). Src family kinases are required for integrin but not PDGFR signal transduction. EMBO J . 18, 2459-2471.

81. Fincham, V., and Frame, M. (1998). The catalytic activity of Src is dispensable for translocation to focal adhesions but controls the turnover of these structures during cell motility. EMBO J . 17, 81-92.

82. Ilic, D., Furuta, Y., Kanazawa, S., Takeda, N., Sobue, K., Nakatsuji, N., Nomura, S., Fujimoto, J., Okada, M., and Yamamoto, T. (1995). Reduced cell motility and enhanced focal adhesion contact formation in cells from FAK-deficient mice. Nature 377, 539-544.

83. Cary, L., Chang, J ., and Guan, J . (1996). Stimulation of cell migration by overexpression of focal adhesion kinase and its association with src and fyn. J . Cell Sci. 109, 1787-1794.

84. Yu, D., Qu, C., Henegariu, O., Lu, X., and Feng, G. (1998). Protein-tyrosine phosphatase Shp-2 regulates cell spreading, migration, and focal adhesion. J. Biol. Chem. 273, 2112521131.

85. Angers-Lousteau, A., Cote, J., Charest, A., Dowbenko, D. Spencer, S., Lasky, L., and Tremblay, M. (1999). Protein tyrosine phosphatase-PEST regulates focal adhesion disassembly, migration, and cytokinesis in fibroblasts. J . Cell Biol. 144, 1019-1031.

86. Schoenwaelder, S. M., and Burridge, K. (1999). Evidence for a calpeptin-sensitive protein-tyrosine phosphatase upstream of the small GTPase Rho. J . Biol. Chem. 20, 14359-14367.

87. Serra-Pages, C., Kedersha, N., Fazikas, L., Medley, Q., De bant, A., and Streuli, M. (1995). The LAR transmembrane protein tyrosine phosphatase and a coiled-coil LAR-interacting protein co-localize at focal adhesions. EMBO J . 14, 2827-2838.

88. Lammer, R., Lerch, M., and Ullrich, A. (2000). The carboxylterminal tyrosine residue of protein-tyrosine phosphatase $\alpha$ mediates association with focal adhesion plaques. J. Biol. Chem. 275, 3391-3396.

89. Yamakita, Y., Totsukawa, G., Yamashiro, S., Fry, D., Zhang X., Hanks, S., and Matsumura, F. (1999). Dissociation of FAK/ p130cas/c-Src complex during mitosis: Role of mitosis-specific serine phosphorylation of FAK. J . Cell Biol. 144, 315-324.

90. Zamir, E., Katz, B., Aota, S., Yamada, K., Geiger, B., and Kam, Z. (1999). Molecular diversity of cell-matrix adhesions. J . Cell Sci. 112, 1655-1669.

91. Katz, B., Zamir, E., Bershadsky, A., Kam, Z., Yamada, K., and Geiger, B. (2000). Physical state of the extracellular matrix regulates the structure and molecular composition of cellmatrix adhesions. Mol. Biol. Cell 11, 1047-1060.

92. Felsenfeld, D., Schwartzberg, P., Venegas, A., Tse, R., and Sheetz, M. (1999). Selective regulation of integrin- cytoskeletal interactions by the tyrosine kinase Src. Nat. Cell Biol. 1, 200-206.

93. Regen, C., and Horwitz, A. F. (1992). Dynamics of betal integrin-mediated adhesive contacts in motile fibroblasts. J . Cell Biol. 119, 1347-1359.

94. Palecek, S., Schmidt, C., Lauffenburger, D., and Horwitz, A. F (1996). Integrin dynamics on the tail region of migrating fibroblasts. J. Cell Sci. 109, 941-952.

95. Smilenov, L., Mikhailov, A., Pelham, R., Marcantonio, E., and Gundersen, G. (1999). Focal adhesion motility revealed in stationary fibroblasts. Science 286, 1172-1174. 
96. Cook, T., Nagasaki, T., and Gundersen, G. (1998). Rho guanosine triphosphatase mediates the selective stabilization of microtubules induced by lysophosphatidic acid. J . Cell Biol . 141, 175- 185.

97. Waterman-Storer, C., Worthylake, R., Liu, B., Burridge, K., and Salmon, E. (1999). Microtubule growth activates Racl to promote lamellipodial protrusion in fibroblasts. Nat. Cell Biol. $1,45-50$.

98. Kaverina, I., Krylyshkina, O., and Small, J . V. (1999). Microtubule targeting of substrate contacts promotes their relaxation and dissociation. J. Cell Biol. 146, 1033-1043.

99. Small, J . V., and Rinnerthaler, G. (1985). Cytostructural dynamics of contact formation during fibroblast locomotion in vitro. Exp. Biol. Med. 10, 54-68.

100. Rinnerthaler, G., Geiger, B., and Small, J . V. (1988). Contact formation during fibroblast locomotion: Involvement of membrane ruffles and microtubules. J. Cell Biol. 106, 747-760.

101. Kaverina, I., Rottner, K., and Small, J . V. (1998). Targeting, capture, and stabilization of microtubules at early focal adhesions. J. Cell Biol. 142, 181-190.

102. Radhakrishna, H., and Donaldson, J . (1997). ADP-ribosylation factor 6 regulates a novel plasma membrane recycling pathway. J . Cell Biol. 139, 49-61.

103. Norman, J ., Barry, S., Holt, M., Cockcroft, A., and Critchley, D. (1998). ARF 1 mediates paxillin recruitment to focal adhesions and potentiates Rho-stimulated stress fiber formation in intact and permeabilized Swiss 3T3 fibroblasts. J. Cell Biol. 143, 1981-1995.

104. Boshans, R., Szanto, S., van Aelst, L., and D'Souza-Schorey, C. (2000). ADP-ribosylation factor 6 regulates actin cytoskel eton remodeling in coordination with Racl and RhoA. Mol. Cell. Biol. 20, 3685-3694.

105. Turner, C., Brown, M., Perrotta, J ., Riedy, M., Nikolopoulos, S., McDonald, A., Bagrodia, S., Thomas, S., and Leventhal, P. (1999). Paxillin LD4 motif binds PAK and PIX through a novel
95-kD ankyrin repeat, ARF-GAP protein: A role in cytoskeletal remodeling. J. Cell Biol. 145, 851- 863.

106. Kondo, A., Hashimoto, S., Yano, H., Nagayama, K., Mazaki, Y., and Sabe, H. (2000). A new paxillin-binding protein, PAG3/ $\mathrm{Pap} \alpha / \mathrm{KI}$ AA0400, bearing an ADP-ribosylation factor GTPase activating protein activity, is involved in paxillin recruitment to focal adhesions and cell migration. Mol. Biol. Cell 11, 13151327.

107. Vitale, N., Patton, W., Moss, J ., Vaughan, M., Lefkowitz, R., and Premont, R. (2000). GIT proteins, a novel family of phosphatidylinositol 3,4,5-trisphosphate-stimulated GTPase-activating proteins for ARF6. J . Biol. Chem. 275, 13901-13906.

108. Fincham, V., Unlu, M., Brunton, V., Pitts, J ., Wyke, J ., and Frame, M. (1996). Translocation of Src kinase to the cell periphery is mediated by the actin cytoskel eton under control of the Rho family of small G proteins. J. Cell Biol. 135, 15511564.

109. Randazzo, P., Andrade, J ., Miura, K., Brown, M., Long, Y.-Q., Stauffer, S., Roller, P., and Cooper, J . (2000). The Arf GTPaseactivating protein ASAP1 regulates the actin cytoskeleton. Proc. Natl. Acad. Sci. USA 97, 4011- 4016.

110. Brown, M., Andrade, J., Radhakrishna, H., Donaldson, J ., Cooper, J ., and Randazzo, P. (1998). ASAP1, a phospholipiddependent ARF GTPase-activating protein that associates with and is phosphorylated by Src. Mol. Cell. Biol. 18, 70387051.

111. Kolanus, W., Nagel, W., Schiller, B., Zeitlman, L., Godar, S., Stockinger, H., and Seed, B. (1996). AlphaL beta2 integrin/ LFA-1 binding to ICAM-1 induced by cytohesin-1, a cytoplasmic regulatory molecule. Cell 86, 233-242.

112. Nagel, W., Zeitlmann, L., Schilcher, P., Geiger, C., Kolanus, J ., and Kolanus, W. (1998). Phosphoinositide 3-OH kinase activates the beta2 integrin adhesion pathway and induces membrane recruitment of cytohesin-1. J . Biol. Chem. 273, 1485314861. 